\title{
Reverse Heat Flow with Peltier-Induced Thermoinductive Effect
}

\section{Kenjiro Okawa (D okawa.k@aist.go.jp )}

National Metrology Institute of Japan, National Institute of Advanced Industrial Science and Technology https://orcid.org/0000-0001-5324-594X

\section{Yasutaka Amagai}

National Metrology Institute of Japan, National Institute of Advanced Industrial Science and Technology https://orcid.org/0000-0001-6816-8158

\section{Hiroyuki Fujiki}

National Metrology Institute of Japan, National Institute of Advanced Industrial Science and Technology Nobu-Hisa Kaneko

National Institute of Advanced Industrial Science and Technology https://orcid.org/0000-0003-38577940

\section{Article}

Keywords: electromagnetic counterparts, thermoinductive, thermal conductivity.

Posted Date: April 9th, 2021

DOI: https://doi.org/10.21203/rs.3.rs-369861/v1

License: (1) (1) This work is licensed under a Creative Commons Attribution 4.0 International License. Read Full License

Version of Record: A version of this preprint was published at Communications Physics on December 1st, 2021. See the published version at https://doi.org/10.1038/s42005-021-00772-4. 


\title{
Reverse heat flow with Peltier-induced thermoinductive effect
}

\author{
Kenjiro Okawa*, Yasutaka Amagai, Hiroyuki Fujiki, and Nobu-Hisa Kaneko \\ National Metrology Institute of Japan, National Institute of Advanced Industrial Science \\ and Technology, 1-1-1 Umezono, Tsukuba, Ibaraki 305-8563, Japan \\ E-mail: okawa.k@aist.go.jp
}

The inductive component is the only missing components in thermal circuits unlike their electromagnetic counterparts. Herein, we report an electrically controllable reverse heat flow, which can be regarded as a proper equivalent of the "thermoinductive" effect. The underlying concept is the heating and cooling of the ends of the material by the Peltier effect under an applied ac current; this form a negative temperature gradient in the opposite direction in a controllable manner. We have derived the exact solution indicating that this reverse heat flow occurs universally in solid-state systems, even in conventional metallic $\mathrm{Cu}$, and that it is considerably enhanced by thermoelectric properties (i.e., a large Seebeck coefficient and low thermal conductivity). A local cooling of $25 \mathrm{mK}$ was demonstrated in $(\mathrm{Bi}, \mathrm{Sb})_{2} \mathrm{Te}_{3}$, which was explained by our exact solution. This electrically controlled reverse heat flow is directly applicable to the fabrication of a "thermoinductor" in thermal circuits. 
Thermal design optimization is an essential issue that needs to be addressed for the development of high-performance electronic devices ${ }^{1-4}$. To date, for the realization of a versatile platform for thermal control in solid-state devices, novel thermal components that can manipulate phonon transport (i.e., thermal $\operatorname{diodes}^{5-7}$, $\operatorname{transistors}^{8,9}$, logic gates $^{10}$, and memories $^{11-13}$ ) have been proposed and discussed ${ }^{14-16}$. These thermal concepts are motivated by the idea of familiar electronic analogs.

An electrical current flow can be essentially replaced with a heat flow by analogy with the Ohm's law 15,17-20; however, an "inductor" is missing among components for thermal circuits $^{15,19}$. This is because oscillatory behaviors, with a reversal in the direction of heat flow from cold to hot, are typically considered violations of the second law of thermodynamics ${ }^{\text {Error! Reference source not found.,21 }}$. An equivalent of the "thermoinductive" effect has been reported in circuits provided with unclear and cumbersome interventions using external heat flows (i.e., natural convection) ${ }^{22,23}$, or the cooling of an electrical coil to the temperature of liquid $\mathrm{He}^{24}$.

Herein, we report an electrically controllable reverse heat flow induced by the Peltier effect with periodic modulation, which operates according to an external ac current, generated in a single thermoelectric (TE) material. The high controllability of heat flow via manipulating the electrical current has promising potential applications. The circuit, composed of a single material, facilitates the understanding of reverse heat flow through theoretical modeling using a simple heat conduction model. Based on the exact solution derived from our heat transfer analysis, we determined an optimized condition to enhance the reverse heat flow occurring in a material. To show proof of concept, we measured and detected the electrical resistance change caused by the reverse heat flow, which is compelling 
evidence for the Peltier-induced thermoinductive effect, achieved using an excellent TE material, $(\mathrm{Bi}, \mathrm{Sb})_{2} \mathrm{Te}_{3}$, near room temperature.

\section{Results}

\section{Modeling of the Peltier-induced thermoinductive effect}

In the rectangular parallelepiped TE material shown in Fig. 1(a), Peltier heating and cooling occur at each interface between the material and the metal electrode under an applied current. Consequently, the direction of the heat flow transmitted as a thermal wave is also reversed with the sign change of the ac current ${ }^{25,26}$. The reversing of the current polarity causes thermal wave collision depending on the position in the material (Fig. 1(b)). An opposite temperature gradient (i.e., a reverse heat flow) can occur in the material at a certain frequency at which the time for reversing the polarity of the ac current is sufficiently faster than the thermal time constant of the material (see the movie included in the Supplementary Information). We derived an exact solution for the heat conduction equation to reveal the temperature distribution in the material.

The temperature rise caused by the Peltier effect $T(x, t)$ at position $x$ and time $t$ is described as

$$
T(x, t)=\frac{S J T_{0}}{\kappa} \frac{1-i}{2 \beta} \frac{e^{(1+i) \beta x}-e^{-(1+i) \beta x}}{e^{(1+i) \beta l / 2}+e^{-(1+i) \beta l / 2}},
$$

where $S, T_{0}, \kappa$, and $l$ are the Seebeck coefficient, mean temperature, thermal conductivity, and length of the sample, respectively. $J$ is the current density given by $J=J_{0} \sin \omega t$ ( $\omega$ is the angular velocity $2 \pi f ; f$ is the current frequency). $\beta \equiv(\omega / 2 \alpha)^{1 / 2}$ is the reciprocal of the thermal diffusion length $D_{\text {th }}=(\alpha / \pi f)^{1 / 2}$, where $\alpha$ is the thermal diffusivity of the sample. $i$ is the imaginary number. The imaginary part corresponds to the phase of the thermal wave. 
Equation (1) is the exact solution for the one-dimensional unsteady-state heat transfer equation (see Methods for calculation details).

Here, we introduce a characteristic dimensionless parameter $F \equiv(l / 2)^{2} f / 2 \alpha$, representing the product of the thermal time constant of the sample $(l / 2)^{2} / \alpha$ and the current frequency. We proposed that $F$ can classify the behavior of the temperature distribution caused by the Peltier heat. Figure 2 shows the relationship between $T(x)$ or heat flow $Q(x)=-\kappa A \mathrm{~d} T(x) / \mathrm{d} x$, where $A$ is the cross-sectional area, and the normalized position $2 x / l . T(x)$ and $Q(x)$ represent the values at the time under the applied root-mean-square (rms) ac current. When the current can be regarded as almost dc current (i.e., $F<<0.05$ ), a linear temperature gradient is caused by the Peltier heating and cooling generated at each sample edge (Figs. 2(a) and (d)). When the ac current of a sufficiently high frequency cancels the TE effect (i.e., $F \gtrsim 15$ ), the temperature distribution is flat, except at the sample edges (Figs. 2(c) and (f)). In contrast, a temperature difference with the opposite direction in the sample occurs when $F \sim 1$ (Figs. 2(b) and (e)). The direction of the temperature gradient is periodically inverted with the polarity reversal of the current (Fig. 1(b)). As can be seen in Fig. 2(h), $Q$ with the opposite (negative) direction occurs near of the sample center $(x \sim 0)$. This uneven heat flow occurring within the material represents a thermal phase delay against the current. This phenomenon can be interpreted in terms of the thermoinductive effect induced by the Peltier effect.

In addition, our calculation based on the exact solution, Eq. (1), shows that while a reverse heat flow can universally occur in any solid material, it is more prominent in TE materials (Fig. 1(c) and (d)). Here, the typical physical properties of $(\mathrm{Bi}, \mathrm{Sb})_{2} \mathrm{Te}_{3}\left(\alpha=1.25 \times 10^{-6} \mathrm{~m}^{2} / \mathrm{s}\right.$, $S=165 \mu \mathrm{V} / \mathrm{K}$, and $\kappa=1.45 \mathrm{~W} / \mathrm{m} \mathrm{K})$ and $\mathrm{Cu}\left(\alpha=1.17 \times 10^{-4} \mathrm{~m}^{2} / \mathrm{s}, S=6.5 \mu \mathrm{V} / \mathrm{K}\right.$, and $\kappa=398 \mathrm{~W} / \mathrm{m} \mathrm{K}$ ) at room temperature were used in the calculation. The negative temperature gradient occurs in both materials, showing a temperature decrease reaching approximately 
$25 \mathrm{mK}$ at $I_{\mathrm{rms}}=10 \mathrm{~mA}$ and $44.4 \mathrm{mHz}$ in $(\mathrm{Bi}, \mathrm{Sb})_{2} \mathrm{Te}_{3}$. A partial temperature inversion of approximately $20 \%$ of the Peltier heating can be achieved. Conventional metallic $\mathrm{Cu}$ shows a temperature decrease of only $3.5 \mu \mathrm{K}$ at $I_{\mathrm{rms}}=10 \mathrm{~mA}$ and $4.16 \mathrm{~Hz}$, which is ten thousand times smaller than that shown by $(\mathrm{Bi}, \mathrm{Sb})_{2} \mathrm{Te}_{3}$. The large Seebeck coefficient is key to enhancing the reverse heat flow. The low thermal conductivity also allows control of the reverse heat flow at low frequencies. However, the directly and high-accuracy detection of minute changes in the heat flow (on the order of $\mu \mathrm{W}$ ) is difficult hindering verification of this phenomenon using general calorimetric measurements.

\section{Electrical resistance changes caused by thermoinductive effect}

We present the analytical model for electrical impedance measurements with a four-probe configuration that can detect temperature changes reflected in the electrical voltage by the Seebeck effect (see Method). The temperature changes caused by the reverse heat flow can be measured as electrical signals on the order of $\mathrm{m} \Omega$ using TE materials. The measured impedance $R$ can be expressed as follows with the ohmic resistance $R_{0}=\rho l_{\mathrm{v}} / A$ :

$$
R=R_{0}\left[1+z T_{0}\left(R_{1}+i R_{2}\right)\right]
$$

where $\rho$ and $l_{\mathrm{v}}$ denote the resistivity and voltage terminal distance. $z$ is the TE figure of merit where $z=S^{2} / \kappa \rho$. Here, $R_{1}$ and $R_{2}$ are the following functions:

$$
R_{1,2}=\frac{\cos \mu \cosh \mu(\sin v \cosh v \pm \cos v \sinh v) \pm \sin \mu \sinh \mu(\sin v \cosh v \operatorname{mcos} v \sinh v)}{2 v\left[(\cos \mu \cosh \mu)^{2}+(\sin \mu \sinh \mu)^{2}\right]},
$$

where $\mu \equiv(2 \pi F)^{1 / 2}$ and $v \equiv \mu l_{\mathrm{v}} / l$ are defined as functions depending on $F$ and $l_{\mathrm{v}} / l$. The second term on the right side in Eq. (2) represents the component resulting from the TE effect, which is increased or decreased by the correction terms $R_{1}$ and $R_{2} . R_{1}$ and $R_{2}$ are considered to correspond with the resistance and reactance components of the TE voltage, respectively. 
Therefore, $R_{1} \rightarrow 1$ and $R_{2} \rightarrow 0$ when $F \rightarrow 0$ as a dc limit, and $R_{1} \rightarrow 0$ and $R_{2} \rightarrow 0$ when $F \rightarrow \infty$ when an ac current with a sufficiently high frequency is applied. The influence of $R_{1}$ and $R_{2}$ can be better observed using high-performance TE materials.

Figures 3(a) and (b) show the calculation results regarding the dependency of the correction terms $R_{1}$ and $R_{2}$ on $F$ obtained using the exact solution of Eq. (3). Here, the physical properties of $(\mathrm{Bi}, \mathrm{Sb})_{2} \mathrm{Te}_{3}$ at room temperature were used. At low current frequencies with $F<10^{-2}, R_{1}$ approaches 1 and the TE effect is sufficiently generated in the sample, but it gradually decreases to 0 as $F$ increases. Notably, as the voltage probe position normalized by the sample length $l_{\mathrm{v}} / l$ becomes smaller, a large dip structure of $R_{1}$ occurs $F \sim 1$. This reflects the situation in which the temperature distribution due to the reverse heat flow shown in Fig. 2(e) is generated in the sample. The dip structure is not measured in the two-probe measurement as $l_{\mathrm{v}} / l=1$. This is consistent with the absence of the dip structure in reports of electrical impedance measurement with the two-probe configuration ${ }^{27}$. Our exact solution revealed that the size of the reverse heat flow varied depending on $x$, and that it could be detected through electrical impedance measurements with changing $l_{\mathrm{v}}$.

To verify the validity of the analytical model, electrical impedance measurements using the TE material $(\mathrm{Bi}, \mathrm{Sb})_{2} \mathrm{Te}_{3}$ were conducted with a four-terminal pair configuration (see Methods for details of the experimental setup). The measurements were performed by changing $l_{\mathrm{v}} / l$ from 0.8 to 0.1 . Figures $3(\mathrm{c})$ and (d) show the frequency dependence of the real and imaginary parts of the measured electrical impedance. The resistance values were gradually decreased with increasing in the current frequency at $l_{\mathrm{v}} / l=0.8$. When $l_{\mathrm{v}} / l=0.5$ and 0.1, a notable dip structure was observed around $40 \mathrm{mHz}$. This frequency region roughly corresponds to $F=1$ in this material. The resistance value for a flat region, corresponding to $R_{0}$, was obtained because the TE effect was sufficiently canceled at frequencies exceeding 
$500 \mathrm{mHz}$. As expected, the calculation results indicated that the size of the dip structure increased with a decreasing $l_{v} / l$. The plots developed using the exact solution, Eq. (2), agreed well with the experimental results over a wide frequency range. The inset of Fig. 3(d) shows the relationship between the normalized resistance and $l_{\mathrm{v}} / l$. At $F=900(f=40 \mathrm{~Hz})$, which is in the flat frequency region in Fig. 3(c), the value of the normalized resistance does not change; this is because of the normalized $R_{0}$, which is approximately 0.65 . The maximum difference from $R_{0}$ is approximately $6.5 \%$ at $l_{\mathrm{v}} / l=0.1 \mathrm{in}(\mathrm{Bi}, \mathrm{Sb})_{2} \mathrm{Te}_{3}$. Conventional metallic $\mathrm{Cu}$ requires detecting minute changes on the order of $\mathrm{n} \Omega$ or less thorough electrical resistivity measurements, which is a challenging task (Fig. S2).

\section{Discussion}

To investigate the conditions under which the reverse heat flow appears, we discussed the heat flow behavior in the frequency domain. In this study, a reverse heat flow is generated by utilizing the material's thermal inertia (the product of the thermal resistivity and volumetric heat capacity) and the phase delay caused by the external current reversal with periodic modulation. Therefore, the behavior of $Q(F)$ can be separable into the resistorcapacitor (RC) component that originates from the thermal inertia and the oscillation component that is induced by the current reversal (Fig. 4). The denominator of the expression of $Q$ (see Eq. (9) in the Method section) corresponds to the RC component as a component independent of $x$ and sharply decreases at $F>0.1$ because of the thermal inertia (the blue dashed line in Fig. 4). In the case of two-probe measurements $\left(l_{\mathrm{v}} / l=1\right)$, the behavior of $Q(F)$ can be explained by considering only this RC component. The component obtained by subtracting the $\mathrm{RC}$ component from $Q(F)$ is responsible for the oscillation of $Q$ with respect to $F$ (the green dashed line in Fig. 4). This oscillation component substantially contributes 
to $Q(F)$ for $F>0.1$, causing a significant reverse heat flow at $F \sim 1$.

In addition, even after $Q(F)$ becomes negative once, $Q(F)$ oscillates with increasing $F$ while repeating its sign inversion. Because the value of $F$ at which the reverse heat flow appears corresponded to that in the case where $Q(F)=0$, the first negative $Q$ appeared in the region of $\pi / 8<F<9 \pi / 8$. Thereafter, the sign of $Q$ inverts in the $\pi$ cycle, which corresponds to the cycle of the current's reversing polarity. Further, we find that when $\mu=(l / 2) / D_{\text {th }}=$ $(2 n-1) \pi / 2,(n=1,2, \ldots)$, the sign of $Q$ is inverted. Therefore, we infer that the reverse heat flow in materials can be controlled by the ratio of the sample length and $D_{\text {th. }}$. This thermoinductive effect is a higher-order thermal response due to the Peltier effect, which cannot be obtained in a conventional lumped-parameter model.

A Peltier-induced reverse heat flow has the advantage of easy tunability using an external current source, in contrast with previously proposed circuits, using external electrical $\operatorname{coils}^{21,24}$ and natural convection ${ }^{22,23}$. For example, the control of heat flow in a fluid system is difficult because the exact analytical model and required experimental conditions are still unclear. Alternatively, the electrical coil with a suitable inductance and internal resistance can be used to adjust the heat flow. In such a case, incorporation of a superconducting coil operating at liquid $\mathrm{He}$ temperatures in the circuit is desirable to reduce the internal resistance of the electrical coil. This impairs the practical implementation of such approaches in thermal circuits. Arbitrary reverse heat flow can be generated without replacing circuit components by tuning the current frequency regardless of the sample size and physical properties (i.e., thermal diffusivity).

In conclusion, we succeeded in analytically formulating and experimentally observing a Peltier-induced reverse heat flow, which can be regarded as an equivalent to the thermoinductive effect, in a single TE material. The exact solution obtained by heat transfer 
analysis clearly demonstrated the optimal conditions for realizing and enhancing this reverse heat flow, induced by the thermal inertia and the phase delay under an external ac current. The excellent agreement between the exact solution and the experiments indicated that the reverse heat flow could be well controlled by the utilization of heat flow with the variablefrequency current. A partial temperature inversion of approximately $20 \%$ of the Peltier heating was achieved at room temperature. These results demonstrate that the utilization of TE materials is a promising strategy for manipulating heat flow in thermal circuits. Our study contributes toward understanding the mechanisms of equivalents of the thermoinductive effect and thereby broadens the scope for thermal circuit design in electronic devices.

\section{Acknowledgments}

The authors are much indebted to Shuji Nakamura, Norihiko Sakamoto and other members of the authors' group in AIST. This work was supported by a Grant-in-Aid for Research Activity Start-up (Grant No.17H07399) from Japan Society for Promotion of Science (JSPS) and partly by JSPS KAKENHI Grant Number JP18H05258.

\section{Author Contributions}

K.O. carried out main experiments and the data analyses. K.O. Y.A., and H.F. developed the concept and conceived experiments. N.-H.K. supervised the project leading to this publication. K.O. wrote the manuscript. All authors contributed to the scientific discussions.

\section{Competing Interests Statement}


The authors declare no competing interests.

\section{Methods}

Experimental setup. Impedance was measured using a polycrystalline p-type $(\mathrm{Bi}, \mathrm{Sb})_{2} \mathrm{Te}_{3}$ material. The dimensions of the sample were $15 \mathrm{~mm} \times 4 \mathrm{~mm} \times 1 \mathrm{~mm}$ as shown in Fig. S3 (see Supplemental information). To capture the Peltier heat generated at the edge of the sample effectively, Au electrodes were fabricated by sputtering. Upon confirming that the adhesion between the sample and the lead wires was insufficient, the measured dc voltage was found to have a large deviation. To suppress the heat conduction of the lead wire, thin Au wires $(\phi=30 \mu \mathrm{m})$ were connected to the sample using Ag paste. To isolate the sample thermally, the sample was suspended with sufficiently long Au wires. The sample space was covered with a radiation shield to reduce heat loss due to thermal radiation. The measurement apparatus was assembled in a vacuum chamber, and measurement was performed under high vacuum conditions $\left(10^{-3} \mathrm{~Pa}\right.$ or less) to suppress the influence of heat convection. The impedance was measured using a commercial impedance analyzer (HIOKI, IM3590). Coaxial cables were used to connect the case, and measurements were performed using the four-terminal pair method. A schematic of the measurement setup in this study is shown in Fig. S3. Although we had to ensure that the Joule heat did not exceed the Peltier heat, the applied current dependency was measured in advance, and the measurement was performed with an rms ac current of $10 \mathrm{~mA}$, which did not affect the results in this study.

Complete derivation of the exact solution. The analysis model in this study is described below. We considered the case where the electrical impedance measurement is performed on 
a rectangular parallelepiped sample with a sample length of $l$ and a cross-sectional area $A$ using the four-terminal pair method with a voltage terminal distance of $l_{\mathrm{v}}$. The Seebeck coefficient, resistivity, thermal conductivity, and thermal diffusivity of the material are denoted as $S, \rho, \kappa$, and $\alpha$, respectively. These physical parameters are considered temperature independent. The temperature distribution at any position $x$ and time $t$ is described as $T(x, t)$. When an ac current with a current density $J=J_{0} \sin \omega t$ is applied, $T(x, t)$ can be obtained by solving the following one-dimensional unsteady-state heat transfer equation ${ }^{28}$ :

$$
\frac{\partial^{2} T(x, t)}{\partial x^{2}}=\frac{1}{\alpha} \frac{\partial T(x, t)}{\partial t}
$$

According to the method of separation of variables, the general solution of Eq. (4) is described as

$$
T(x, t)=C_{1} e^{ \pm(1+i) \beta x-i \omega t}+C_{2} e^{\mathrm{m}(1+i) \beta x-i \omega t},
$$

where $C_{1}$ and $C_{2}$ are arbitrary constants. From Eq. (5), it can be understood that $T(x, t)$ behaves as a thermal wave. The imaginary part corresponds to the phase of the thermal wave. For simplicity, we assume that only Peltier heat $Q_{\text {Peltier }}=S J T_{0}$ occurs at both ends of the sample $x= \pm l / 2$, where $T_{0}$ is the mean temperature of the sample. Assuming that all the Peltier heat flows into the sample, the effects of other heat losses, such as those by convection and radiation, are not considered in this model. Then, the boundary condition is described as follows:

$$
\left.\frac{\partial T(x, t)}{\partial x}\right|_{x= \pm l / 2}=\frac{S J T_{0}}{\kappa} .
$$

Under this boundary condition, the arbitrary constants of the general solution of Eq. (5) are obtained. Then, $T(x, t)$ is given by Eq.(1). The behaviors of $T(x)$ and $Q(x)$ versus $F$ are described in detail in Fig. S1. 
Using $T(x, t)$ obtained from Eq. (1), the voltage measured between $\pm l_{\mathrm{v}} / 2$ is expressed as follows:

$$
V=l_{\mathrm{v}} \rho J+\int_{-l / 2}^{l / 2} S \frac{\partial T(x, t)}{\partial x} d x
$$

The first term on the right side represents the ohmic voltage between $l_{\mathrm{v}}$, and the second term represents the Seebeck voltage. In actual measurements, the temperature rise caused by the Peltier effect is considered small $(\sim 0.1 \mathrm{~K})$, so the integral part can be replaced with $2 S T\left(x=l_{\mathrm{v}} / 2, t\right)$. The measured impedance $R$ in Eq. (2) can be introduced from $R=J / A$.

A heat flow $Q=-\kappa A \mathrm{~d} T(x, t) / \mathrm{d} x$ is described as follows:

$$
Q=-J S T_{0} A \frac{e^{(1+i) \beta x}+e^{-(1+i) \beta x}}{e^{(1+i) \beta l / 2}+e^{-(1+i) \beta l / 2}}
$$

The real part of $Q$ can be rewritten as follows:

$$
\operatorname{Re}(Q)=-J S T_{0} A \frac{\cos \beta x \cosh \beta x \cos \mu \cosh \mu+\sin \beta x \sinh \beta x \sin \mu \sinh \mu}{\cos ^{2} \mu \cosh ^{2} \mu+\sin ^{2} \mu \sinh ^{2} \mu}
$$

The imaginary part of $Q$ corresponds to the phase of the heat flow.

In the experimental setup used in this study, only small temperature changes of less than $0.1 \mathrm{~K}$ occurred in the TE material. To increase this effect, the current value can be increased by using a TE material with high $z T_{0}$. However, our model ignores the influence of Joule heating; therefore, if the current value is increased, the effect of Joule heating cannot be ignored. In such a case, a more complicated analysis is required.

\section{References}

1 Garimella, S. V., Fleischer, A. S., Murthy, J. Y., Keshavarzi, A., Prasher, R., Patel, C., 
Bhavnani, S. H., Venkatasubramanian, R., Mahajan, R., Joshi, Y., Sammakia, B., Myers, B. A., Chorosinski, L., Baelmans, M., Sathyamurthy, P., \& Raad, P. E. Thermal challenges in next-generation electronic systems. IEEE Trans. Components Packag. Technol. 31, 801 (2008).

2 Ma, K., He, N., Liserre, M. \& Blaabjerg, F. Frequency-domain thermal modeling and characterization of power semiconductor devices. IEEE Trans. Power Electron. 31, 7183 (2016).

3 Ahmed, H. E., Salman, B. H., Kherbeet, A. Sh. \& Ahmed, M. I. Optimization of thermal design of heat sinks: A review. Int. J. Heat Mass Tranf. 118, 129 (2018).

4 Shen, Y., Wang, H., Blaabjerg, F., Zhao, H., \& Long, T. Thermal modeling and design optimization of PCB vias and pads. IEEE Trans. Power Electron. 35, 882 (2020).

5 Li, B., Wang, L., \& Casati, G. Thermal diode: Rectification of heat flux. Phys. Rev. Lett. 93, 184301 (2004).

6 Ben-Abdallah, P., \& Biehs, S. A. Phase-change radiative thermal diode. Appl. Phys. Lett. 103, 191907 (2013).

7 Martínez-Pérez, M. J., Fornieri, A., \& Giazotto, F. Rectification of electronic heat current by a hybrid thermal diode. Nat. Nanotechnol. 10, 303 (2015).

8 Li, B., Wang, L., \& Casati, G. Negative differential thermal resistance and thermal transistor. Appl. Phys. Lett. 88, 143501 (2006).

9 Sood, A., Xiong, F., Chen, S., Wang, H., Selli, D., Zhang, J., McClellan, C. J., Sun, J. Donadio, D., Cui, Y., Pop,E., \& Goodson, K. E. An electrochemical thermal transistor. Nat. Commun. 9, 4510 (2018).

10 Wang, L., \& Li, B. Thermal Logic Gates: Computation with phonons. Phys. Rev. Lett. 99, 177208 (2007). 
11 Wang, L., \& Li, B. Thermal memory: A storage of phononic information. Phys. Rev. Lett. 101, 267203 (2008).

12 Kubytskyi, V., Biehs, S.-A., \& Ben-Abdallah, P. Radiative bistability and thermal memory. Phys. Rev. Lett. 113, 074301 (2014).

13 Guarcello, C., Solinas, P., Braggio, A., Ventra, M. D., \& Giazotto, F. Josephson thermal memory. Phys. Rev. Applied 9, 014021 (2018).

14 Li, N., Re, J., Wang, L., Zhang, G., Hanggi, P., \& Li, B. Colloquium: Phononics: Manipulating heat flow with electronic analogs and beyond. Rev. Mod. Phys. 84, 1045 (2012).

15 Wehmeyer, G., Yabuki, T., Monachon, C., Wu, J., \& Dames, C. Thermal diodes, regulators, and switches: Physical mechanisms and potential applications. Appl. Phys. Rev. 4, 041304 (2017).

16 Ye, H., Leung, S. Y. Y., Wong, C. K. Y., Lin, K., Chen, X., Fan, J., Kjelstrup, S., Fan, X., \& Zhang, G. Thermal inductance in GaN devices. IEEE Electron Device Lett. 37, 1473 (2016).

17 Bosworth, R. C. L. The thermal Ohm, Farad and Henry. Philos. Mag. 37, 803 (1946).

18 Weedy, B. M. The analogy between thermal and electrical quantities. Elector. Pow. Syst. Res. 15, 197 (1988).

19 Lienhard, J. H. V, \& Lienhard, J. H. IV, A Heat Transfer Textbook: Fifth Edition (Dover Books on Engineering), (2019).

20 Piggott, A. J., \& Allen, J. S. Peltier supercooling with isosceles current pulses: A response surface perspective. ECS J. Solid State Sci. Technol. 6, N3045 (2017).

21 Bossen, O. \& Schilling, A. LC-circuit calorimetry. Rev. Sci. Instrum. 82, 094901 (2011).

22 Bosworth, R. C. L. Thermal inductance. Nature 158, 309 (1946).

23 Bosworth, R. C. L. Thermal mutual inductance. Nature 161, 166 (1948). 
24 Schilling, A., Zhang, X. \& Bossen, O. Heat flowing from cold to hot without external intervention by using a "thermal inductor". Sci. Adv. 5, eaat9553 (2019).

25 Iwasaki, H., \& Hori, H. Thermoelectric property measurement by the improved Harman method. in International Conference on Thermoelectrics, ICT Proceedings, pp.501 (2005).

26 Downey, A. D., Hogan, T. P., \& Cook, B., Characterization of thermoelectric elements and devices by impedance spectroscopy. Rev. Sci. Instrum. 78, 093904 (2007).

27 García-Cañadas, J., \& Min, G., Low frequency impedance spectroscopy analysis of Thermoelectric Modules. J. Electron. Mater. 43, 2411 (2014).

28 Kirby, C. G. M., \& Laubitz, M. J. The error due to the Peltier effect in direct-current measurements of resistance. Metrologia 9, 103 (1973). 

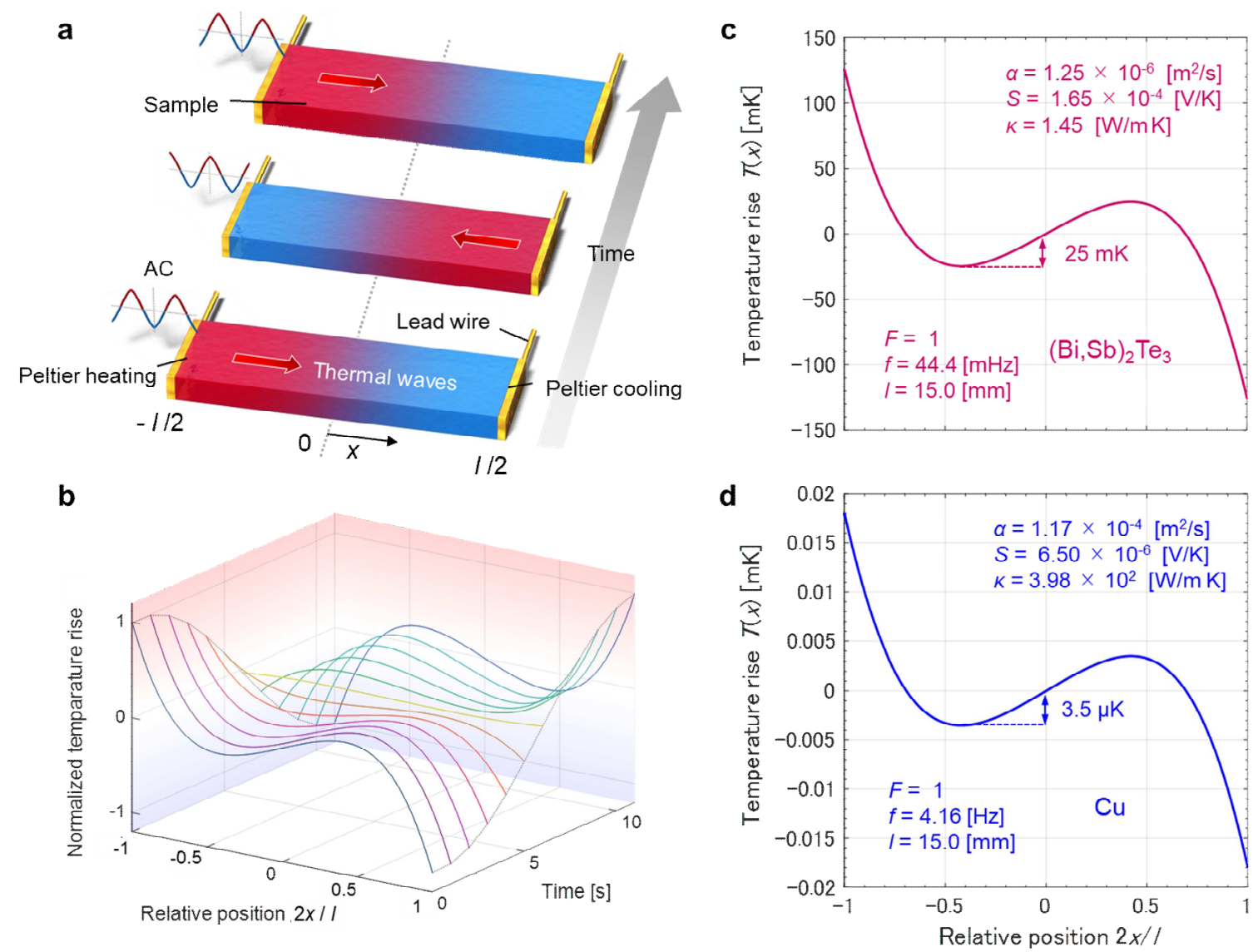

Figure 1 | Temperature change caused by the Peltier effect with periodic modulation.

(a) Schematic of changes in temperature of the material. By applying an ac current, Peltier heating and cooling are generated at the edge of the sample and the thermal oscillation propagates in the material as a thermal wave. The direction of the wave is changed by reversing the polarity of the ac current. (b) Simulation of the temperature rise caused by the Peltier effect with periodic modulation at the edge of the sample as a function of relative position in the sample $2 x / l$ and time in the case of the dimensionless parameter $F=1$ (see the text). The vertical axis is normalized by the value at the edge, $2 x / l=-1$. Calculation results based on the exact solution of the temperature rise $T(x)$ dependence of $2 x / l$ at the time under the applied root-mean-square (rms) ac current in (c) a TE material (Bi,Sb) $\mathrm{Te}_{3}$ and (d) conventional metallic $\mathrm{Cu}$ for $F=1$. Typical physical properties of $(\mathrm{Bi}, \mathrm{Sb})_{2} \mathrm{Te}_{3}(\alpha=1.25$ 
$\times 10^{-6} \mathrm{~m}^{2} / \mathrm{s}, S=165 \mu \mathrm{V} / \mathrm{K}$, and $\left.\kappa=1.45 \mathrm{~W} / \mathrm{m} \mathrm{K}\right)$ and $\mathrm{Cu}\left(\alpha=1.17 \times 10^{-4} \mathrm{~m}^{2} / \mathrm{s}, S=6.5 \mu \mathrm{V} / \mathrm{K}\right.$, and $\kappa=398 \mathrm{~W} / \mathrm{m} \mathrm{K}$ ) at room temperature were used in the calculation. 


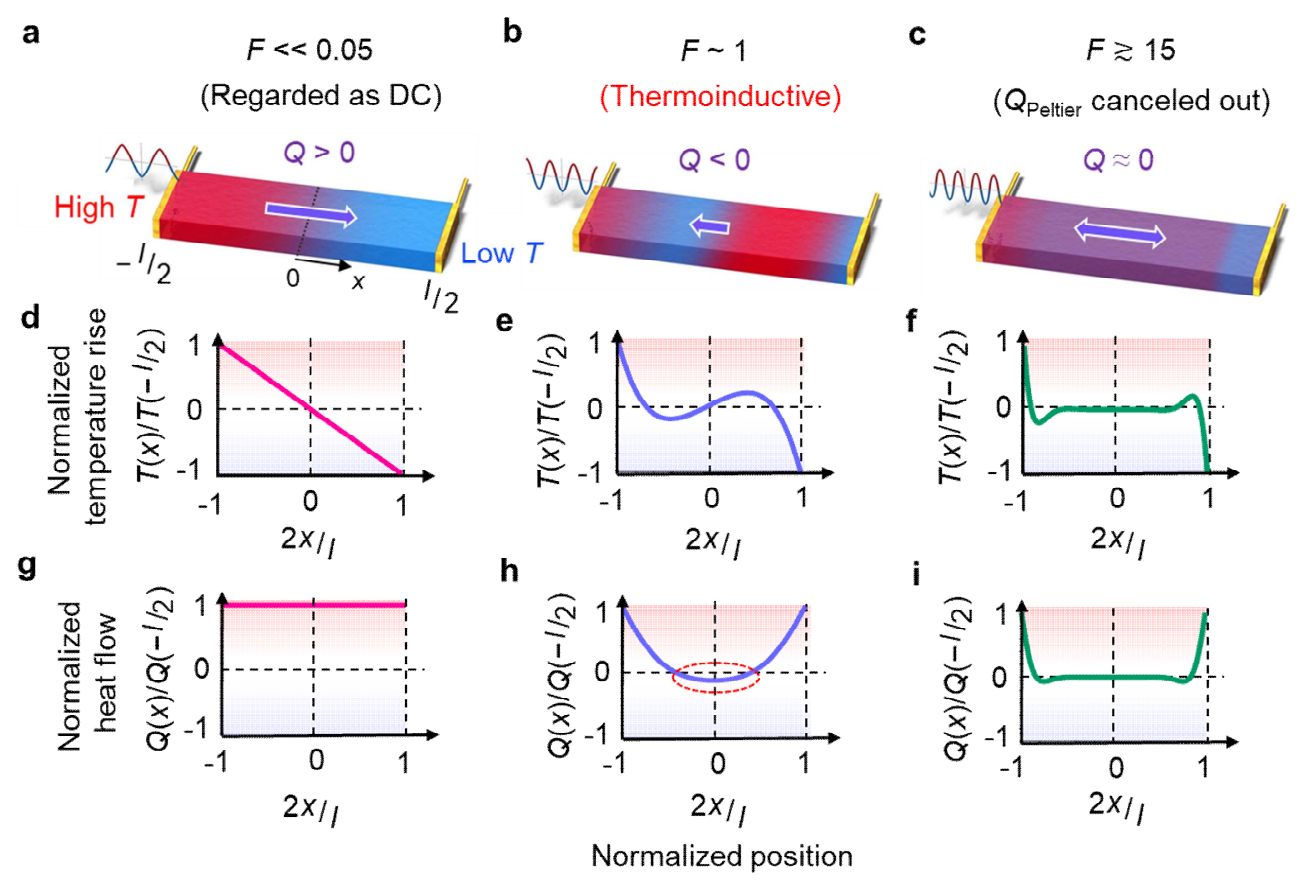

Figure 2 | Classification of the temperature distribution and heat flow induced by Peltier effect.

(a)-(c) Schematic of the temperature distribution $T(x)$ and heat flow $Q(x)$ in the sample at the time under the applied rms ac current. The relationship (d)-(f) between $T(x)$ and relative position in the sample $2 x / l$, and (g)-(i) between $Q(x)$ and $2 x / l$. Each situation is classified by the characteristic dimensionless parameter $F<<0.05, \sim 1$, and $\gtrsim 15$. In the case of $F \sim 1$, the thermoinductive effect (reverse heat flow) occurs because of the Peltier effect. 
a

b
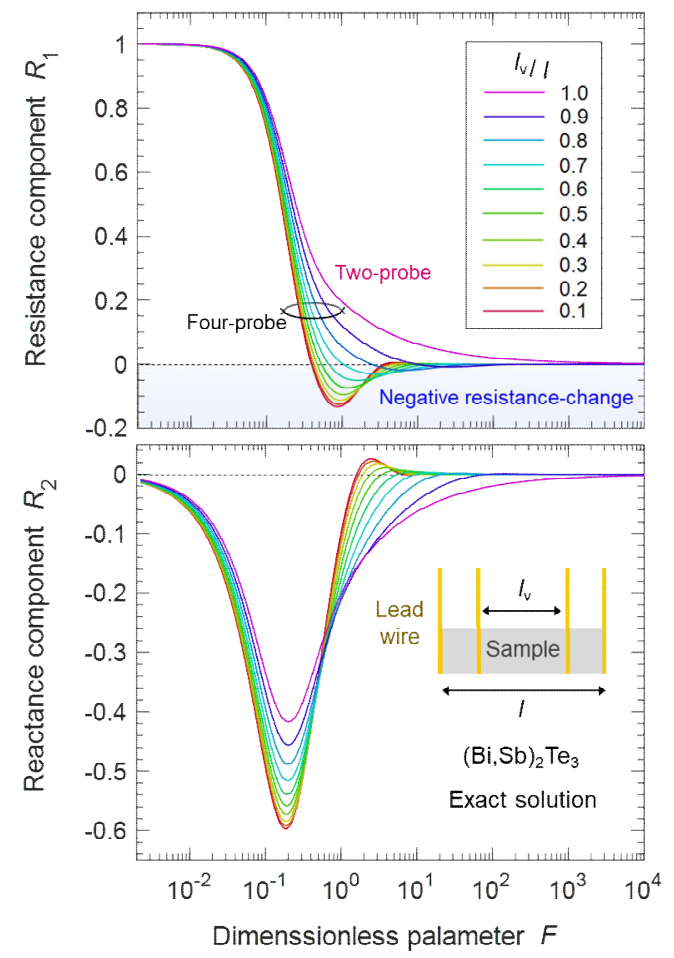

C
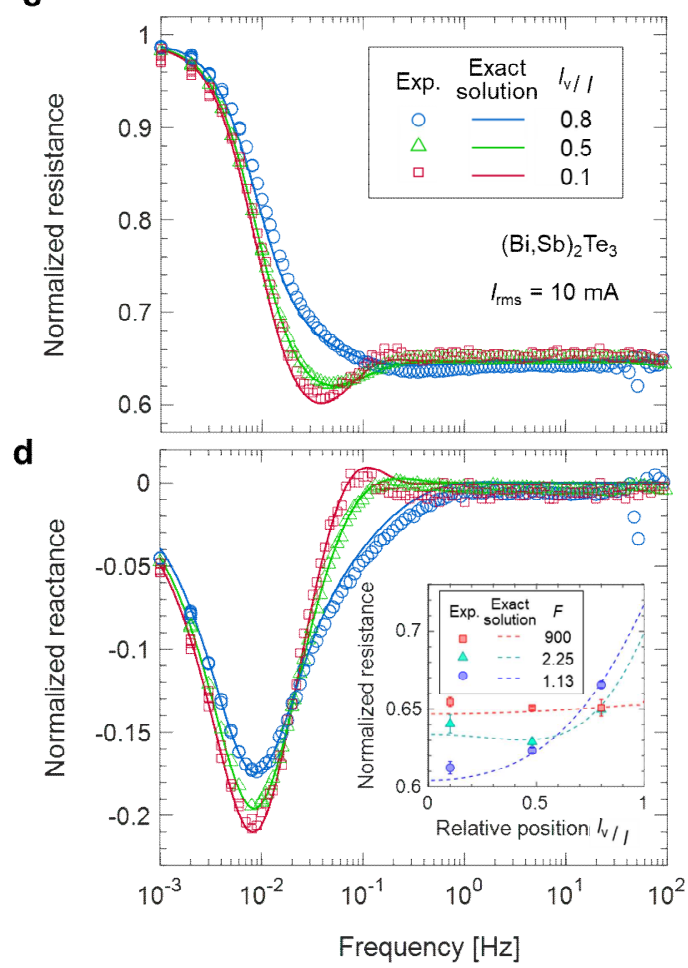

Figure 3 | Calculation and experimental results of Peltier-induced thermoinductive

effect.

Calculation results based on the exact solution regarding the dependence of characteristic dimensionless parameter $F$ on the correction terms (a) $R_{1}$ and (b) $R_{2}$ using $(\mathrm{Bi}, \mathrm{Sb})_{2} \mathrm{Te}_{3}$ with $\alpha=1.25 \times 10^{-6} \mathrm{~m}^{2} / \mathrm{s}, S=166 \mu \mathrm{V} / \mathrm{K}, \rho=1.1 \times 10^{-5} \Omega \mathrm{m}, \kappa=1.45 \mathrm{~W} / \mathrm{m} \mathrm{K}$, and $l=15 \mathrm{~mm}$. The voltage probe position normalized by the sample length $l_{\mathrm{v}} / l$ was varied from 0.1 to 1.0 . Inset in (b) shows the illustration of the four-probe configuration. Experimental results of frequency dependence of the (c) real and (d) imaginary parts of the impedance of $(\mathrm{Bi}, \mathrm{Sb})_{2} \mathrm{Te}_{3}$. Open symbols are experimental results, and solid lines are calculation results based on the exact solution, Eq. (2). Red circles, green triangles, and purple squares denote the experimental results corresponding to $l_{\mathrm{v}} / l=0.8,0.5$, and 0.1 , respectively. The vertical axis is normalized by the value for the dc measurement $(F \rightarrow 0)$. The inset shows the relationship 
between the normalized resistance and $l_{\mathrm{v}} / l$. Dotted lines are calculation results. Red squares, green triangles, and blue circles are the experimental results corresponding to $F=900,2.25$, and 1.13 , respectively. 


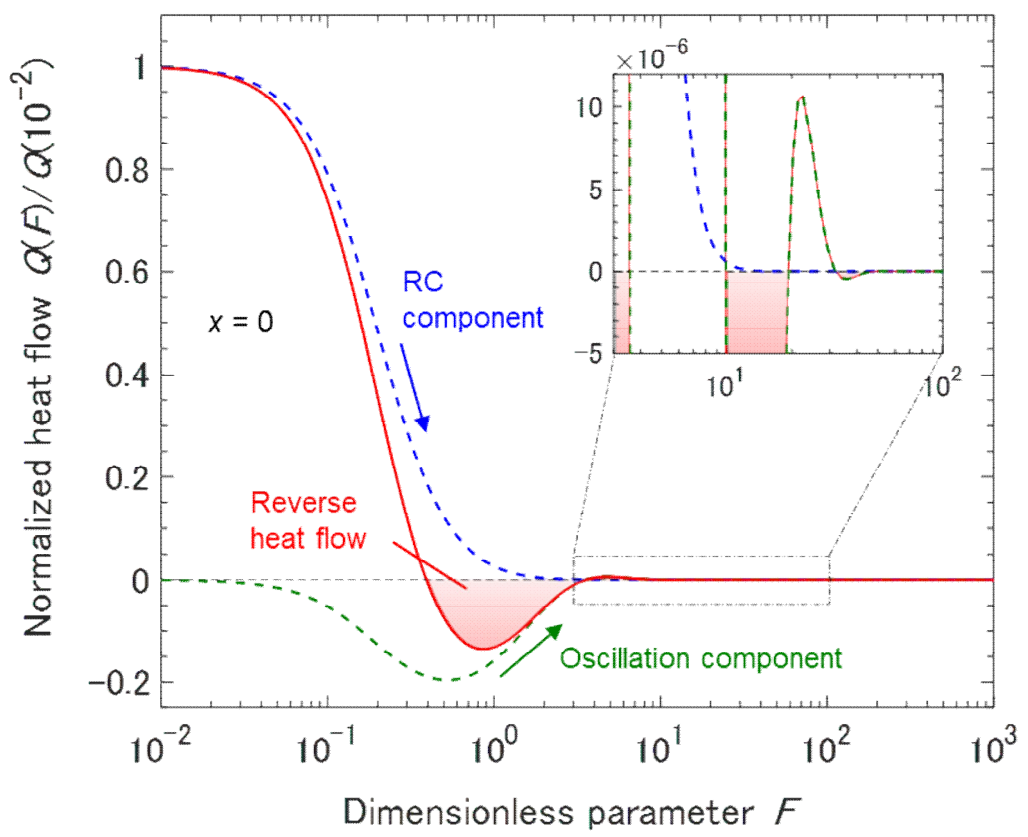

Figure $4 \mid F$ region in which reverse heat flow $(Q<0)$ is expected.

Calculation results showing dependence of dimensionless parameter $F$ on the heat flow $Q(F)$ at $x=0$. The vertical axis is normalized by the value at $F=10^{-2}$. The solid red line is the heat flow $Q(F)$ calculated using Eq. (9). The blue dotted line is the attenuation term of $Q(F)$, corresponding to the $\mathrm{RC}$ component towing to the material's thermal inertia. The green dotted line is the oscillation term of $Q(F)$, corresponding to the oscillation component induced by the external current reversal. The inset shows an enlarged view of the damped oscillation in the larger $F$ region. 


\section{Figures}
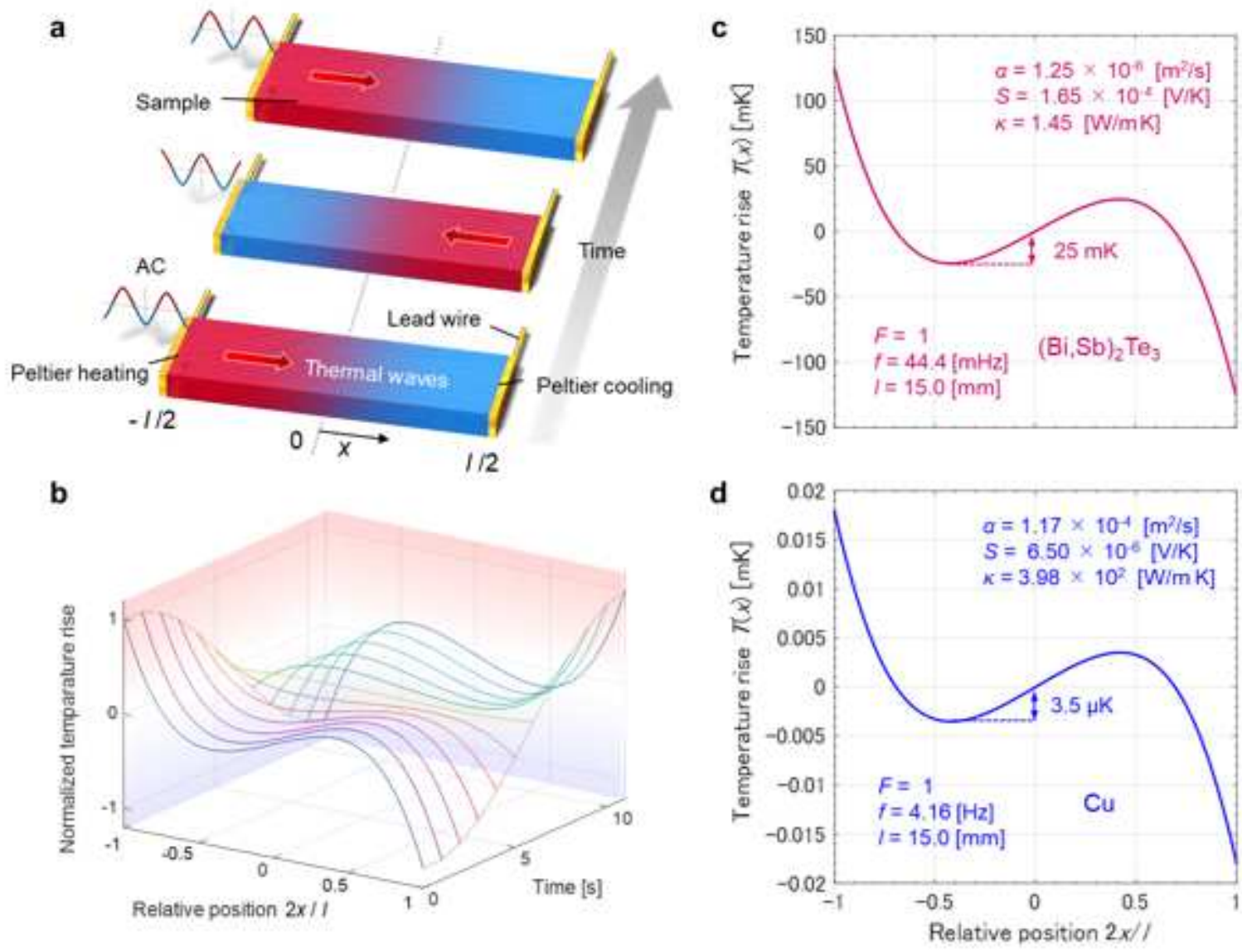

\section{Figure 1}

Temperature change caused by the Peltier effect with periodic modulation. (a) Schematic of changes in temperature of the material. By applying an ac current, Peltier heating and cooling are generated at the edge of the sample and the thermal oscillation propagates in the material as a thermal wave. The direction of the wave is changed by reversing the polarity of the ac current. (b) Simulation of the temperature rise caused by the Peltier effect with periodic modulation at the edge of the sample as a function of relative position in the sample $2 x / I$ and time in the case of the dimensionless parameter $F=1$ (see the text). The vertical axis is normalized by the value at the edge, $2 x / I=-1$. Calculation results based on the exact solution of the temperature rise $T(x)$ dependence of $2 x / I$ at the time under the applied rootmean-square (rms) ac current in (c) a TE material (Bi,Sb)2Te3 and (d) conventional metallic Cu for $\mathrm{F}=1$. Typical physical properties of $(\mathrm{Bi}, \mathrm{Sb}) 2 \mathrm{Te} 3(\mathrm{a}=1.25 \times 10-6 \mathrm{~m} 2 / \mathrm{s}, \mathrm{S}=165 \mu \mathrm{V} / \mathrm{K}$, and $\mathrm{K}=1.45 \mathrm{~W} / \mathrm{m} \mathrm{K})$ and $\mathrm{Cu}(\mathrm{a}=1.17 \times 10-4 \mathrm{~m} 2 / \mathrm{s}, \mathrm{S}=6.5 \mu \mathrm{V} / \mathrm{K}$, and $\mathrm{K}=398 \mathrm{~W} / \mathrm{m} \mathrm{K})$ at room temperature were used in the calculation. 
a $F \ll 0.05$ (Regarded as DC)
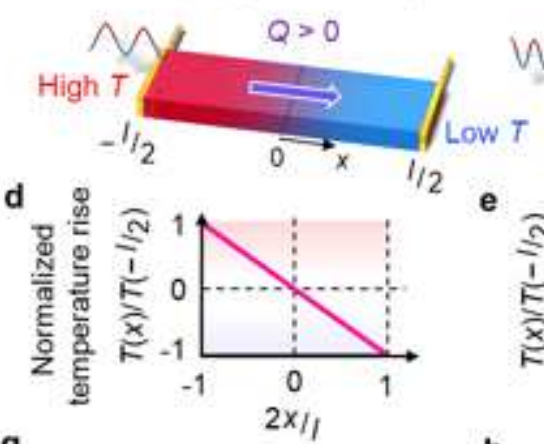

g

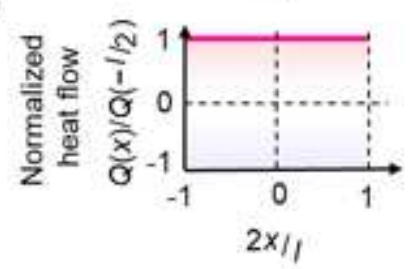

b

$F \sim 1$

(Thermoinductive)

Whe $Q<0$

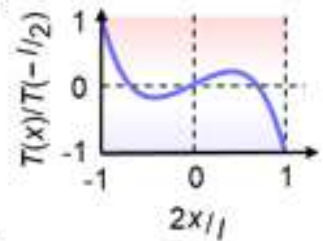

h

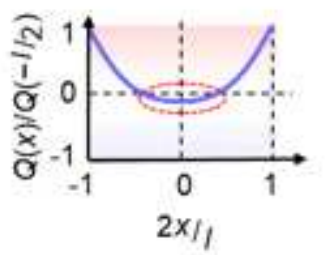

Normalized position c

$F \gtrsim 15$

( $Q_{\text {peiter }}$ canceled out)
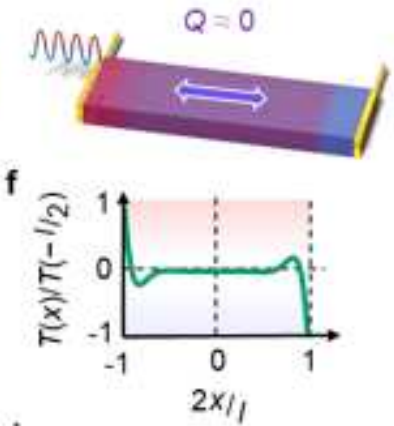

i

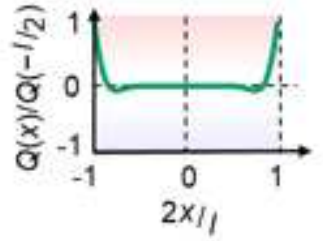

Figure 2

Classification of the temperature distribution and heat flow induced by Peltier effect. (a) - (c) Schematic of the temperature distribution $\mathrm{T}(\mathrm{x})$ and heat flow $\mathrm{Q}(\mathrm{x})$ in the sample at the time under the applied rms ac current. The relationship (d) - (f) between $T(x)$ and relative position in the sample $2 x / l$, and $(g)-(i)$ between $\mathrm{Q}(\mathrm{x})$ and $2 \mathrm{x} / \mathrm{l}$. Each situation is classified by the characteristic dimensionless parameter $\mathrm{F}<<0.05, \sim 1$, and $\otimes 15$. In the case of $F \sim 1$, the thermoinductive effect (reverse heat flow) occurs because of the Peltier effect. 
a

b

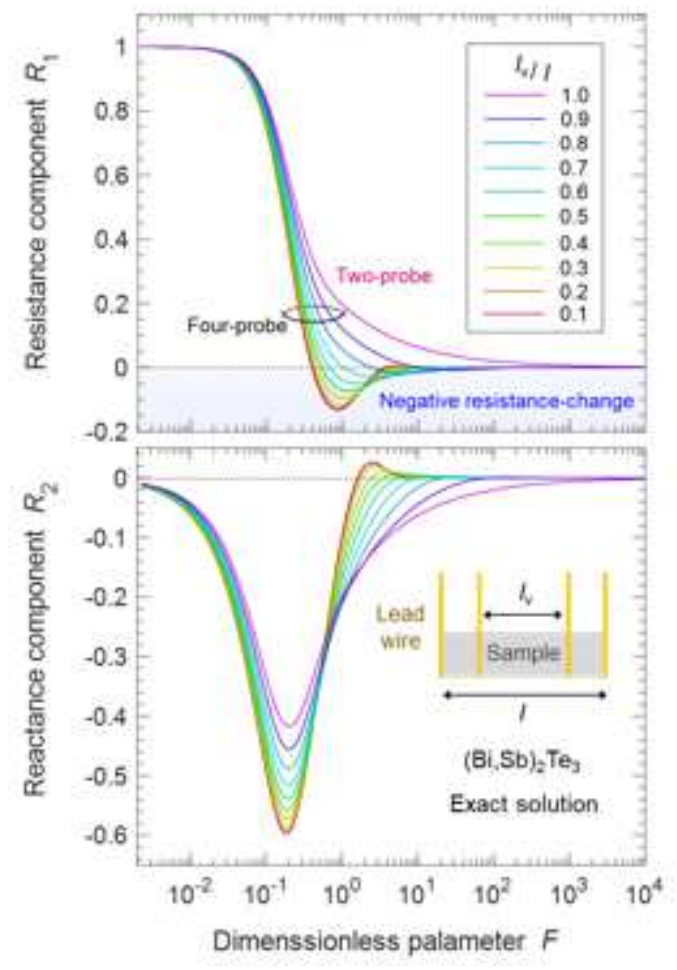

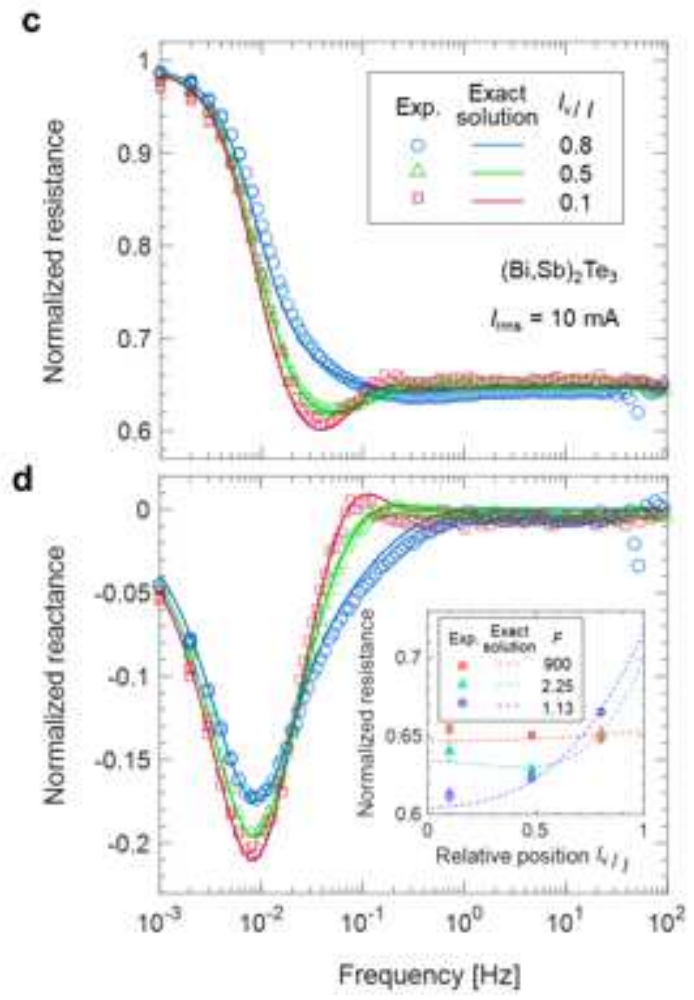

Figure 3

Calculation and experimental results of Peltier-induced thermoinductive effect. Calculation results based on the exact solution regarding the dependence of characteristic dimensionless parameter $\mathrm{F}$ on the correction terms (a) R1 and (b) R2 using (Bi,Sb)2Te3 with $\mathrm{a}=1.25 \times 10-6 \mathrm{~m} 2 / \mathrm{s}, \mathrm{S}=166 \mu \mathrm{V} / \mathrm{K}, \rho=1.1 \times$ $10-5 \Omega \square \mathrm{m}, \mathrm{K}=1.45 \mathrm{~W} / \mathrm{m} \mathrm{K}$, and I $=15 \mathrm{~mm}$. The voltage probe position normalized by the sample length Iv/I was varied from 0.1 to 1.0. Inset in (b) shows the illustration of the four-probe configuration.

Experimental results of frequency dependence of the (c) real and (d) imaginary parts of the impedance of $(\mathrm{Bi}, \mathrm{Sb}) 2 \mathrm{Te} 3$. Open symbols are experimental results, and solid lines are calculation results based on the exact solution, Eq. (2). Red circles, green triangles, and purple squares denote the experimental results corresponding to Iv $/ \mathrm{I}=0.8,0.5$, and 0.1 , respectively. The vertical axis is normalized by the value for the dc measurement (Fख0). The inset shows the relationship between the normalized resistance and Iv/l. Dotted lines are calculation results. Red squares, green triangles, and blue circles are the experimental results corresponding to $F=900,2.25$, and 1.13 , respectively. 


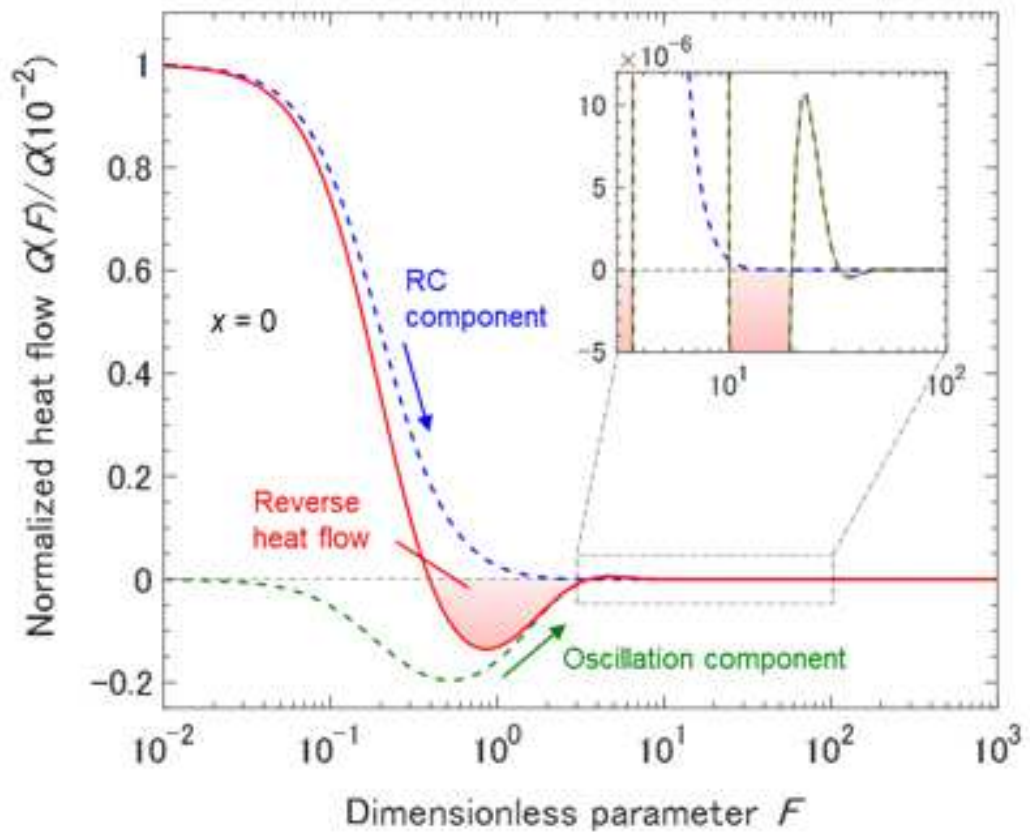

Figure 4

$F$ region in which reverse heat flow $(Q<0)$ is expected. Calculation results showing dependence of dimensionless parameter $F$ on the heat flow $Q(F)$ at $x=0$. The vertical axis is normalized by the value at $F$ $=10-2$. The solid red line is the heat flow $Q(F)$ calculated using Eq. (9). The blue dotted line is the attenuation term of $\mathrm{Q}(\mathrm{F})$, corresponding to the $\mathrm{RC}$ component towing to the material's thermal inertia. The green dotted line is the oscillation term of $Q(F)$, corresponding to the oscillation component induced by the external current reversal. The inset shows an enlarged view of the damped oscillation in the larger $F$ region. 